\title{
FRANCINE DESCARRIES, SOCIOLOGUE-MILITANTE FÉMINISTE QUÉBÉCOISE. INSTITUTIONNALISER LES ÉTUDES FÉMINISTES POUR TRANSFORMER LA SOCIÉTÉ
}

\author{
Entretien réalisé par Véronica Gomes, Marie Mathieu
}

Éditions Antipodes | « Nouvelles Questions Féministes »

2019/2 Vol. 38 | pages 108 à 121

ISSN 0248-4951

ISBN 9782889011674

Article disponible en ligne à l'adresse :

https://www.cairn.info/revue-nouvelles-questions-feministes-2019-2-page-108.htm

Distribution électronique Cairn.info pour Éditions Antipodes.

(C) Éditions Antipodes. Tous droits réservés pour tous pays.

La reproduction ou représentation de cet article, notamment par photocopie, n'est autorisée que dans les limites des conditions générales d'utilisation du site ou, le cas échéant, des conditions générales de la licence souscrite par votre établissement. Toute autre reproduction ou représentation, en tout ou partie, sous quelque forme et de quelque manière que ce soit, est interdite sauf accord préalable et écrit de l'éditeur, en dehors des cas prévus par la législation en vigueur en France. Il est précisé que son stockage dans une base de données est également interdit. 


\title{
arcours
}

\section{Francine Descarries, sociologue-militante féministe québécoise Institutionnaliser les études féministes pour transformer la société}

\author{
Entretien réalisé par Véronica Gomes et Marie Mathieu
}

Francine Descarries est professeure de sociologie depuis 1985 à l'Université du Québec à Montréal (UQAM). Auteure en 1980 d'un des premiers ouvrages québécois sur la reproduction sociale des sexes, L'école rose... et les cols roses ${ }^{1}$, issu de son mémoire de maîtrise, ses travaux de recherche portent sur les théories féministes, l'évolution du discours et des pratiques du mouvement des femmes québécois, de même que sur la famille, le travail des femmes et la reproduction de la division sociale des sexes. Aujourd'hui figure de proue du féminisme québécois, elle a largement contribué à faire reconnaître la légitimité scientifique des recherches et études féministes au Québec et ouvré pour leur assurer une plus grande présence au sein de l'université. Membre fondatrice de l'Institut de recherches et d'études féministes (IREF) de l'UQAM, elle a été, tour à tour, directrice académique de l'Alliance de recherche IREF - Relais-femmes (ARIR, de 2000 à 2010) et directrice scientifique du Réseau québécois en études féministes (RéQEF, de 2011 à 2019). Après avoir été récompensée en 2011 du Prix d'excellence en recherche et création de l'Université du Québec (volet carrière) et en 2012 du Prix Ursula Franklin pour l'étude du genre de la Société Royale du Canada, elle a coordonné et assuré la direction du comité scientifique du septième Congrès international des recherches féministes dans la francophonie (CIRFF) qui a eu lieu à Montréal en 2015. Ayant été toutes deux ses étudiantes et ses

1. L'école rose... et les cols roses. La reproduction de la division sociale des sexes, Centrale de l'enseignement du Québec, 1980. 
collègues, nous sommes heureuses de laisser une trace dans NQF de l'engagement inépuisable de Francine en faveur des études féministes.

Véronica Gomes: Tout d'abord nous voudrions que tu reviennes sur ta trajectoire étudiante et professionnelle. Quelles en sont les étapes les plus importantes qui ont conduit à ton positionnement féministe dans le monde universitaire?

Francine Descarries: J'ai dû interrompre mes études à l'âge de 16 ans, car mon père est décédé et ma famille n'avait les moyens de payer les études que d'une seule personne, or il m'apparaissait évident - comme à ma famille - que ce soit mon frère. Ce qui me frappe quand je reviens sur cette situation, c'est à quel point je l'acceptais. J'ai pleuré durant vingt-quatre heures, puisqu'on venait de court-circuiter mon rêve de continuer mes études classiques au collège Marie-France pour devenir travailleuse sociale ou avocate. Ça a été un vrai drame, mais un drame que j'ai accepté très rapidement, car à cet âge, j'étais bien inscrite dans le modèle traditionnel et bien adaptée à ce qu'on attendait de moi. J'ai donc abandonné mon projet d'études et suis devenue secrétaire légale, ce qui était tout de même bien pour une fille à l'époque. Après deux années à ce poste, on m'a offert l'opportunité d'être agente de voyage, ce que j'ai fait pendant sept ans. Cela m'a permis de voir le monde et d'acquérir une indépendance dès mes 18 ans. À la naissance de ma première fille, je me suis aperçue alors que ce métier, qui demandait de nombreux déplacements, ne s'articulait pas très bien avec mes nouvelles responsabilités maternelles. Du moins je le croyais à l'époque. J'ai donc démissionné et suis restée à la maison jusqu'à la naissance de ma seconde fille. Mais rapidement, j'ai réalisé que je ne pourrais me contenter du rôle de mère au foyer et que j'avais besoin de stimulations extérieures. Je suis donc retournée aux études à 27 ans avec l'appui de mon conjoint.

Je suis, de ce point de vue, un pur produit de la Révolution tranquille puisque j'ai repris mes études en 1969, soit un an après la création des Cégeps $^{2}$, ce qui me permettait d'accéder à des études pré-universitaires gratuites offrant une promotion par matière. Assez vite, j'ai opté pour une orientation sciences humaines avec mathématiques, bien que l'orienteur du collège m'ait dit que je devrais probablement abandonner ce projet parce qu'une femme de mon âge (je vous rappelle que j'avais alors 27 ans) pourrait avoir de la difficulté à comprendre ce qu'on appelait alors les «maths nouvelles". Le Cégep a été un passage relativement facile et surtout très formateur, puisque j'ai commencé à m'intéresser à la situation des femmes

2. Les Cégeps ou Collèges d'enseignement général et professionnel sont des établissements publics assurant l'enseignement pré-universitaire au Québec. Les études collégiales font suite à l'enseignement en école secondaire. 
et que j'y ai consacré la plupart de mes travaux de recherche, à l'exception peut-être d'un en histoire qui portait sur l'industrie de la pêche en Nouvelle-France. Je n'avais pas vraiment d'antécédents féministes et ce choix n'a pas été fait de façon réfléchie. Il s'est imposé à moi progressivement et je me suis assez rapidement passionnée pour le féminisme et définie comme féministe. J'aimerais bien pouvoir dire que Simone de Beauvoir a été mon inspiration et que j'ai trouvé ma voie en lisant son œuvre, mais non! C'est plutôt en lisant La Femme mystifiée ${ }^{3}$ de Betty Friedan que j'ai compris le trouble qui m'habitait alors, parce que le "malaise des femmes" qu'elle décrivait correspondait assez bien à ma propre expérience. Ce qui m'était apparu normal jusque-là, soit me définir à travers mon rôle d'épouse et de mère, ne faisait soudainement plus sens. Et puis, il y a eu Ainsi soit-elle de $^{4}$ Benoîte Groult, qui a été pour moi un vrai coup de massue. J'ai réalisé alors que l'identité et le rôle des femmes - et donc qui j'étais - nous étaient assignés par des normes et des attentes externes sur lesquelles nous n'avions aucun contrôle. J'ai progressivement pris conscience des effets discriminatoires de ce que je sais maintenant être la division sexuelle du travail. Mais à l'époque, nous commencions à peine à nommer ce rapport de pouvoir qui était alors totalement naturalisé et intégré dans nos vies. Notre lot pour "gagner le ciel", comme on disait autrefois, c'était finalement une condition d'asservissement à l'autorité maritale et à l'ensemble des institutions politiques patriarcales.

Mes études de baccalauréat ${ }^{5}$ à l'Université de Montréal (UdM) ont été un non-événement, même si à chaque fois que j'ai pu, j'ai effectué mes travaux sur les femmes et leurs conditions de vie. C'est comme ça que tranquillement je me suis dirigée vers les études de maîtrise et que j’ai décidé de faire un mémoire sur les femmes, en me posant une question fondamentale, que je me pose toujours quarante ans plus tard: comment expliquer que les femmes acceptent d'occuper les places et les positions qui leur sont assignées socialement? Quels sont les mécanismes à l'œuvre? Comment se reproduisent-ils? Et comment peut-on expliquer que ces impositions soient incorporées dans le vécu des femmes et dans leur rapport au monde? À l'époque, l'approche marxiste prédominait. Dans mon mémoire de mâ̂trise, j'ai réellement mis des gants blancs pour expliquer que j'allais transformer cette grille de lecture et proposer une analyse en termes de rapports sociaux, mais cette fois en termes de rapports sociaux de sexe et de division sexuelle du travail. J'ai fait ce mémoire avec Louis Maheu, un directeur extraordinaire, qui m'a appris à faire de très longs titres avec des deuxpoints [sourire]. J'ai surtout découvert avec lui l'importance de la synthèse

3. Paru en anglais en 1963 sous son titre originel The Feminine Mystique, cet essai a été traduit en français en 1964.

4. Paru en 1975.

5. Le baccalauréat correspond au premier cycle universitaire au Québec. 
et d'une pensée organisée. En retour, j'ai sans doute été la première à lui proposer de diriger un mémoire féministe. Il faut dire qu'en 1978, moment où je terminais ma maîtrise, Isabelle Grémy [Lasvergnas], Zaîda Radja et moi-même avons été les premières à initier, à titre de chargées de cours, l'enseignement de "Sociologie de la condition des femmes" qui s'est donné à l'UdM. L'expérience a duré deux années, puis nous avons été remplacées par Nicole Laurin et Danièle Juteau.

Pour le doctorat, j'ai décidé de poursuivre mes études à l'Université de Montréal. Ne voyant pas vraiment qui, à ce moment-là, aurait pu me diriger en sociologie féministe, j'ai saisi une chance qui m'était offerte: devenir chargée de projet dans le cadre d'une recherche sur le développement de la science au Québec et de l'activité de publication des biologistes et des psychologues québécois francophones, ainsi que continuer ma thèse avec le même directeur. Je me doutais bien que je ne resterais pas toute ma vie dans ce champ d'études, mais l'encadrement offert m'assurait un apprentissage méthodologique, analytique et conceptuel très solide. Dans ma thèse ${ }^{6}, \mathrm{j}^{\prime}$ ai montré que les scientifiques qui consacraient plus de temps au développement du champ scientifique québécois étaient ceux dont les carrières étaient les moins reconnues et les moins visibles sur le plan international. C'est assez intéressant, car cela fait résonance avec ma propre trajectoire. Ma thèse avait pour objectif d'analyser la manière dont les périphéries travaillent et surtout de montrer comment le français intervient dans la construction du savoir et des carrières scientifiques. Déjà en 1980, il était évident que les scientifiques qui publiaient en français freinaient leur évolution professionnelle. Néanmoins, j’ai pris la décision de publier mes textes en français, mais dans mon cas, c'était en toute connaissance de cause. Si j'allais au doctorat, c'était avec l'intention bien nette de devenir professeure d'université, j'ai été chanceuse à cet égard, car mon doctorat en poche, j'ai été embauchée à l'UQAM, à la toute fin des vagues d'embauches du début des années 1980. Par la suite, la situation est devenue très difficile et les postes se sont fait beaucoup plus rares pendant pratiquement une décennie.

Véronica: Dans quelle mesure tes expériences personnelles et professionnelles - notamment extra-universitaires - ont nourri tes choix d'objets de recherche?

Je me pose souvent cette question. J'aimerais être capable de situer le jour de mon épiphanie, soit le moment où j'aurais compris que je devais penser et agir comme féministe. Ce qui est certain, c'est que lorsque je suis arrivée

6. Descarries-Bélanger, Francine (1984). Production des savoirs spécialisés et développement de la science au Québec: l'activité de publication des biologistes et des psychologues québécois francophones. Thèse présentée à la Faculté des études supérieures en vue de l'obtention du grade de Ph. D., Université de Montréal. 
à l'UQAM en 1985, c'était la période des dernières grandes batailles pour l'avortement libre et gratuit, des premiers questionnements sur les technologies de la reproduction, des premières études sur les stéréotypes sexuels, de l'arrivée massive des femmes et des mères sur le marché du travail, etc. Comme je l'ai mentionné, c'est vraiment progressivement que j'en suis venue à me questionner sur les expériences des femmes et sur le féminisme. Je suis revenue aux problèmes que j'avais soulevés et laissés en suspens dans mon mémoire de maîtrise. Les choses n'avaient pas beaucoup changé depuis la publication de l'ouvrage qui en était issu. J'avais toujours les mêmes questions. Il était alors évident pour moi qu'il me fallait appréhender le rapport des femmes à la société, à la maternité, à leur identité, en interprétant celuici à partir d'un point de vue situé et en reconnaissant que ces rapports portaient l'empreinte d'une dynamique imposée à travers différents types de violence symbolique et matérielle qui ne relevait en rien de l'ordre de la nature. J'ai voulu savoir ce que pensaient et disaient les femmes de leurs expériences. À cette époque, c'était complètement nouveau et marginalisé. Je m’intéressais aussi à ce que disait le féminisme sur les femmes et sur les inégalités entre femmes et hommes. Plus tard, je me suis penchée sur les rapports sociaux de sexe, la division et la hiérarchisation des groupes de sexe: quelle lecture du rapport hommes-femmes faisaient les féministes et quelles stratégies étaient pensées et développées selon chacune des problématiques rencontrées? C'est là que j'ai commencé à travailler sur les différents courants de pensée du féminisme contemporain, d'abord avec Shirley Roy alors étudiante au doctorat, avec qui j'ai publié un premier essai de typologie des courants de pensée dans le mouvement des femmes ${ }^{7}$. Cet essai m'a permis d'entrer en contact avec beaucoup de féministes à travers le Canada, l'ouvrage ayant été traduit en anglais.

Puis, le champ de mes intérêts de recherche s'est élargi à la suite d'une rencontre avec Christine Corbeil, alors professeure au département de travail social. Elle est venue me voir un jour et m'a dit: «J'aimerais travailler sur la maternité avec toi." J'ai dit oui parce que cela venait aussi me chercher dans ma vie personnelle. Je crois avoir toujours été une bonne épouse et une bonne mère de famille. J'ai adoré cela, sans jamais me sentir aliénée... mais mon expérience était en dissonance avec l'analyse féministe que j'en faisais, surtout à partir du moment où j'ai vraiment adopté comme référent théorique le féminisme matérialiste. Quand j'ai travaillé sur la typologie des courants féministes, j'ai pu voir comment j'étais passée d'un féminisme très égalitariste et réformiste à un féminisme beaucoup plus contestataire. Sur le plan personnel, ma vie a toujours été satisfaisante, mais parfois moins radicale que mes réflexions et peut-être un peu plus proche de la vie des femmes et de leurs expériences de tous les jours. J'étais alors, et je suis encore, dans 
un dilemme théorique et personnel entre maternité-relation et maternité-institution qui renvoient, j'en suis consciente, à des dimensions contradictoires d'une même expérience. L'ancrage de ma lutte repose sur la conviction que la maternité ne doit en aucun cas être imposée et que l'on ne doit pas priver les femmes de projets de vie personnels ou légitimer leur appropriation. Dans la foulée des travaux entrepris avec Christine Corbeil, j'ai cherché à savoir comment les féministes ont pensé la maternité. J'ai donc refait un travail de typologie, mais en regardant cette fois-ci comment les différents courants de pensée considéraient la maternité et quels étaient les enseignements à tirer de leurs différentes propositions. C'était important pour moi, je voulais introduire l'expérience maternelle au cœur de la réflexion et des pratiques féministes car j'étais profondément convaincue que le féminisme n'avait de sens que s'il était un agent de changement. Qu'on le veuille ou non, la maternité demeure un fait social qu'expérimente la grande majorité des femmes de la planète et constitue, à mon avis, le nœud gordien du devenir femmes.

Marie Mathieu : Tu as eu au cours de ta carrière universitaire différentes casquettes: féministe militante, enseignante, chercheuse, directrice de I'ARIR puis du RéQEF, épouse, mère et aujourd'hui tu es une grand-mère très présente dans la vie de sa petite-fille. Comment as-tu réussi à articuler ces différentes activités tout au long de ton parcours?

Je commencerais par dire qu'ont compté la santé, l'énergie, la capacité de dormir mais aussi celle de relativiser les choses. Ce sont des essentiels. J'ai souvent pensé à cela. Il aurait suffi, par exemple, que j'aie une enfant malade ou que je sois dans une situation économique précaire pour ne pas avoir la même carrière. Dans mon cas, je pense que ces essentiels se sont conjugués à ma volonté d'inscrire les études féministes comme un champ de savoir susceptible de modifier le monde et les rapports sociaux dans leur ensemble, mais aussi à ma détermination à imposer les perspectives féministes dans l'analyse des faits sociaux. Cela a été les moteurs de mon action. Je suis comblée par la réussite de ma famille, par mes relations avec mes enfants et mon conjoint et par mon travail d'enseignante, mais il y a des zones du travail intellectuel où j'aurais aimé être plus performante. Cela étant, je suis tout de même assez satisfaite de mon parcours académique, même si je n'ai pas gagné sur tous les fronts. Je mentirais si je disais que j'ai réussi l'articulation famille-travail, ne serait-ce qu'au niveau du partage de la charge mentale. C'était tellement nouveau cette idée de retour aux études pour une femme mariée, que je m'étais convaincue que rien ne devait changer dans ma vie familiale. J'assumais toutes les tâches pour ne pas déranger l'harmonie de cette famille basée sur la division sexuelle du travail. J'ai réussi mes études en étant mère de deux enfants, en travaillant à plein temps, en rognant très souvent sur mes heures de sommeil, en veillant tard et en me levant tôt. Après mon embauche comme professeure, j'ai pu bénéficier de 
l'aide d'une personne - aujourd'hui une amie - qui vient chez moi deux fois par semaine depuis trente-cinq ans. Je me suis beaucoup reposée sur elle. Et puis, il y a à peine une dizaine d'années, lorsque ma fille aînée a eu un accident, j'ai décidé de prendre un temps d'arrêt pour m'occuper d'elle. Je me suis alors aperçue que tout pouvait attendre et que tout était relatif. Je me suis fixé un nouvel ordre de priorité. C'est sûr que si j’avais été un homme, j'aurai bien plus investi ma carrière et discuté sociologie pendant bien plus d'heures, mais cela ne me manque pas.

Véronica: Pourquoi préfères-tu le terme d'articulation famille-travail à celui de conciliation?

En fait, l'articulation c'est un terme que nous avons tenté d'imposer avec Christine Corbeil, mais nous n'avons pas réussi. Pour nous, concilier veut dire coping, soit "faire avec». Ce n'est pas possible de faire avec et ce n'est pas aux femmes de faire avec. Dans une certaine mesure, la conciliation famille-travail fait appel à la responsabilité individuelle des femmes: comment nous nous organisons pour être des mères travailleuses heureuses. Selon nous, c'est un faux départ. La preuve: trente ans plus tard, on pense encore plus souvent à comment faciliter la vie des femmes, sans poser la véritable question, soit celle de la division sexuelle du travail et des pratiques qui en découlent. Si les femmes ont fait énormément de progrès dans l'espace public, les hommes n'en ont pas fait autant dans l'espace privé... Je pense qu'ils ont réellement modifié leur rapport à la paternité sous l'influence des requêtes féministes, mais ils ne partagent pas encore suffisamment la charge mentale inhérente à la gestion familiale, pas plus qu'ils ne s'inscrivent de manière égalitaire dans la routine domestique. Bref, pour nous, concilier voulait dire mettre ensemble deux univers que nous avions posés comme inconciliables, il reste donc à voir comment les articuler.

Marie: Tu dis souvent qu'être féministe c'est vivre avec ses contradictions. Qu'est-ce que tu entends par là?

Nous avons été socialisées à notre condition de femme, nous avons incorporé la vision du féminin et de la féminité, nous avons appris la sollicitude dans notre rapport aux autres. Qu'on le veuille ou non, aujourd'hui encore, les femmes sont formées pour prendre soin des autres et des jugements sont portés sur celles qui se distancient de ce qui est donné comme leur devoir naturel. Vivre avec ses contradictions, c'est savoir cela et essayer de faire autrement, mais c'est aussi accepter qu'il puisse parfois exister une distance entre ce qu'on dénonce en théorie et ce qu'on pratique dans les faits. Je me suis promenée dans les manifestations avec cette pancarte: "Ni pape, ni 
juge, ni médecin, ni conjoint. C'est aux femmes de décider!», mais si j’avais dû avorter, j'aurais sans doute senti le besoin de négocier avec mon conjoint. Même si j'adhère totalement au principe que l'avortement est une décision qui appartient aux femmes, dans la pratique je ne l'aurais probablement pas prise toute seule. Au quotidien, c'est un ensemble de petites choses qui te ramène constamment à des rôles que tu sais que tu n'as pas à adopter, mais qui, dans une certaine mesure, font partie de toi. C'est peut-être vieux jeu, mais ces contradictions surviennent souvent au regard du contrat éthique qui t'engage auprès de certains êtres humains.

Être féministe, ce n'est pas seulement pour moi vivre des contradictions, c'est aussi, comme le disait Gloria Steinem, être en colère pratiquement tous les jours, dès que tu ouvres ton journal notamment. C'est ne pas rester indifférente face à toutes les situations inéquitables que vivent les femmes. C'est aussi se sentir impuissante face à celles-ci et se questionner sur l'impact de sa contribution en tant qu'intellectuelle. Ti-Grace Atkinson disait que "penser les femmes" et théoriser leurs expériences est une action militante en soi. Cette phrase a été très importante pour moi parce qu'elle m'a permis de me dédouaner en partie et de donner un sens à ma pratique de professeure-chercheure.

Véronica: Pourquoi as-tu décidé de t'engager dans l'institutionnalisation des études féministes?

Pourquoi l'institutionnalisation? Parce qu'il m'apparaissait que les femmes étaient victimes d'un préjudice épistémique total et que leurs réalités étaient gommées. Je m’intéressais au travail, mais ce dernier n'était pas pensé dans des termes qui incluaient les femmes. Le travail des femmes était perçu alors comme complémentaire, avec la notion de salaire d'appoint particulièrement présente. À la fin de ma thèse, j'étais très consciente que si je voulais travailler en études féministes, c'était à l'UQAM qu'il fallait que je le fasse. Il y avait alors Micheline de Sève, Évelyne Tardy, Louise Vandelac, Yolande Cohen, Nadia Fahmy-Eid, Karen Messing, pour ne nommer qu'elles, qui étaient là. Pour moi, ces femmes ouvraient vraiment des voies de réflexion : femmes et politique, femmes et histoire, femmes et rapports sociaux de sexe... alors que, dans le milieu universitaire, la réflexion féministe, voire sur les femmes, tardait à émerger au Québec, en France et même aux ÉtatsUnis. Le savoir était vraiment en construction à ce moment-là. Il y avait bien des ouvrages importants, mais on les comptait sur les cinq doigts de la main. Shulamith Firestone, Kate Millett, Ann Oakley nous ont beaucoup influencées. Beaucoup plus que les Françaises, au départ.

Les études féministes au début des années 1980 demeuraient très marginales. Il fallait donc sortir ce champ de sa périphérie et travailler à 
l'institutionnaliser. Les collègues du GIERF $^{8}$ avaient déjà mis en place les premiers cours en études féministes, et ce depuis 1972. Donc, quand je suis arrivée à l'UQAM, il y avait déjà une identité féministe qui se développait et cette identité me rejoignait et m'inspirait. Elle a nourri mes espoirs d'une activité professionnelle où je pourrais travailler avec et sur les femmes. Pour pouvoir bien y travailler, personnellement comme collectivement, il fallait développer un milieu accueillant et capable de promouvoir le développement des études féministes ainsi que de produire des nouveaux savoirs. Il fallait donc institutionnaliser les études féministes à l'UQAM, ce qui, pour moi, a passé essentiellement par trois étapes. La première a été la création de l'IREF. Initié par Louise Vandelac, ma collègue du département de sociologie de l'UQAM, le projet a été porté collectivement par quelques membres enthousiastes du GIERF dont j'étais. Ensuite, à travers deux projets d'Alliance de recherche IREF-Relais-femmes (ARIR), j'ai pu obtenir, de 2000 à 2010, des financements conséquents pour stimuler la réalisation de nombreux projets de recherche et favoriser des activités de transfert et de valorisation des connaissances dans les milieux universitaire et communautaire. Enfin, au regard des résultats obtenus par l'ARIR, j'ai cru possible de développer un Réseau québécois en études féministes, interdisciplinaire et interuniversitaire (RéQEF). Depuis 2011, grâce à une subvention des Fonds de recherche société et culture, le RéQEF est venu concrétiser un rêve, que certaines d'entre nous, telles que Maria de Koninck, Huguette Dagenais, Micheline Beauregard, Marielle Tremblay et Anita Caron, transportions depuis au moins une vingtaine d'années. À deux ou trois reprises, pendant ces années, nous avions essayé de construire un réseau, mais sans grand succès, faute de ressources et ne pouvant compter que sur le bénévolat des membres et sur la possibilité de nous retrouver au congrès de l'ACFAS ${ }^{9}$, une fois l'an. Aujourd'hui le RéQEF est présent dans douze institutions universitaires québécoises et regroupe près de trois cents membres, dont plus d'une centaine de membres régulières, près de vingt chercheur.e.s des milieux de pratique et près de cent cinquante membres étudiant·e·s aux cycles supérieurs.

Véronica : Puis il y a eu le Congrès international des recherches féministes dans la francophonie, en 2015 : était-ce dans la lancée du RéOEF qu'il a été organisé à Montréal?

Oui, c'était une conséquence assez logique. Puisque l'IREF se portait bien et que le RéQEF venait d'émerger, constituant un important réseau de

8. Le GIERF est le groupe interdisciplinaire de recherche et d'études féministes, créé en 1976, qui peut être considéré comme l'ancêtre de l'IREF à l'UQAM.

9. L'ACFAS est l'Association francophone pour le savoir, anciennement désignée Association canadienne-française pour l'avancement des sciences. Elle organise chaque année depuis 1933 un congrès scientifique, multidisciplinaire et interdisciplinaire. 
chercheur-e.s universitaires, il était évident que nous ne pouvions pas passer à côté de l'idée d'organiser un Congrès international des recherches féministes (CIRFF) à Montréal, ne serait-ce que pour faire valoir la présence francophone. Parce que, comme vous le savez, les Québécoises vivent dans un certain isolement, enclavées dans un univers anglophone. Les études féministes, on doit bien l'admettre, se développent pour l'essentiel en anglais. Cela implique une façon de voir les choses, un mode de lecture particulier. Nous nous sommes aperçues très rapidement que les matérialistes françaises dont les travaux commençaient à nous parvenir et qui nous offraient un nouveau vocabulaire pour comprendre les rapports sociaux de sexe étaient très peu lues, hors du Québec et, par conséquent, très peu citées. Or, dans notre univers, ce n'est pas simplement publish or perish, mais il faut aussi être citée pour subsister! Nous avons donc rapidement accueilli cette perspective féministe francophone et cherché à la faire connaître.

Pour moi, les CIRFF doivent servir à créer des liens entre chercheures francophones et à s'inspirer les unes et les autres. C'est une pensée qui reste largement inconnue dans le milieu anglophone notamment canadien et qui n'arrive pas à avoir l'impact analytique et politique qu'elle devrait avoir. Je pense que les jeunes générations de nouvelles enseignantes et chercheures renforceront cet échange entre la France et le Québec. Nos programmes en études féministes ont produit des théoriciennes et des enseignanteschercheures féministes de haut niveau qui, contrairement à ma génération, auront les espaces et les occasions d'échange pour travailler, se spécialiser et avoir un apport qui sera plus international que le nôtre.

Véronica: Tu travailles et tu vis quotidiennement avec différentes générations de femmes et de féministes: tes filles, ta petite-fille, tes collègues de travail mais aussi les étudiantes qui participent à tes cours. Comment ces relations contribuent à ta réflexion sur le féminisme, à ton féminisme et à ta façon de le transmettre?

Grosse question parce qu'effectivement, très souvent, ces rapports m'amènent dans des espaces de contradictions. D’abord les étudiantes m'interpellent, certaines me déconcertent par l'importance qu'elles accordent à la corporéité, à l'identité sexuelle et à l'agentivité individuelle, des analyses qui gomment pour partie le fait social que constituent les rapports sociaux de sexe. En même temps, elles me forcent à me questionner et à diriger mon regard ailleurs. La plus belle chose dans l'enseignement, c'est l'obligation de recevoir les idées, les problématiques et les préoccupations des plus jeunes, même si je trouve quelquefois difficile de les accepter. Pour le moins, cela me force à me tenir le plus possible à jour sur les tendances actuelles du féminisme. 
Parallèlement, depuis une dizaine d'années environ, a ressurgi dans le milieu universitaire un militantisme qui me rapproche beaucoup plus de celui que nous pratiquions dans les années 1980. Certaines personnes s'en plaindront, mais moi, je côtoie des étudiantes et des jeunes femmes qui veulent faire une différence, qui pensent souvent très différemment de moi, mais qui pensent merveilleusement bien. Je considère avoir une chance exceptionnelle d'être restée assez longtemps dans le milieu universitaire pour voir à l'œuvre des générations de féministes que nous formons ou avons formées ici à l'UQAM. Qu'elles soient professeures ou étudiantes en maîtrise ou au doctorat, leur réflexion est riche et ouverte sur une perspective intersectionnelle.

Mes filles et ma petite-fille, quant à elles, me procurent beaucoup de bonheur, car j'aime mon rôle actuel de mère et de grand-mère. Mes filles sont toutes les deux féministes dans leurs pratiques, sans en tenir nécessairement le discours, qu'elles ont laissé à leur mère. Elles l'ont assez entendu pour l'avoir bien intériorisé. Par contre, j'avoue que ma petitefille me fait bien plaisir lorsque, à 13 ans, elle se déclare féministe et choisit comme sujet de recherche la féministe québécoise Idola Saint-Jean, se disant très fière que sa grand-mère en ait suivi les traces. Notre relation me procure énormément de joie, car elle me permet de rattraper des retards que j'ai accumulés à l'époque où je travaillais sur mes thèses, alors que mes enfants avaient le même âge que ma petite-fille.

Marie: Quelle place tes collaborations avec les organismes communautaires et plus largement le travail collectif ont-elles eu dans ton cheminement intellectuel et professionnel?

Mon cheminement avec les groupes de femmes est intéressant parce que j'ai l'impression que ce sont elles qui m'ont permis de mieux exprimer ma pensée féministe. Dans ma pratique, cela a été l'occasion d'échanges fructueux et de transferts mutuels de savoirs. Ma collaboration avec les groupes de femmes m'a amenée à travailler, à différents moments, sur des problématiques précises que je n'aurais peut-être pas abordées autrement. Cela a donné un aspect plus concret, plus stratégique à ma pratique. Les femmes de ces groupes m'ont stimulée, elles m'ont animée et je pense qu'en retour, j'ai pu aussi leur permettre quelquefois de mieux comprendre certaines réalités et de se familiariser avec les différents lexiques de la pensée féministe. Elles m'ont amenée à travailler sur la publicité sexiste, sur les exploitations sexuelles, sur la participation des créatrices dans différents milieux culturels, comme sur l'antiféminisme par exemple. Avec elles, la même question revenait souvent: comment contrer les mécanismes qui empêchent les femmes d'exploiter leur plein potentiel? 
Marie: Récemment, dans une interview, tu t'es définie comme une "féministe structuro-matérialiste consubstantielle radicale et égalitariste en faveur d'une sociologie collective ${ }^{10}$. Peux-tu revenir sur ce que cela signifie pour toi?

Ce que je voulais mettre de l'avant, par cette formule, c'est que pour arriver à construire une pensée féministe et des stratégies d'intervention, il faut faire du bricolage. Je pense que c'est une erreur de dire: "Je suis de tel ou tel courant». La majorité des luttes a été gagnée par un féminisme égalitariste et nous avons eu besoin de ces luttes. Nous avions besoin de meilleures opportunités, d'un salaire égal pour un travail égal, de garderies, d'accéder à l'autodétermination de nos corps et de nos sexualités, de lutter pour briser le plafond de verre et de nous dégager du plancher collant... Sans le féminisme égalitariste, il n'y aurait pas eu ces batailles. Mais en même temps, se déclarer féministe égalitariste, c'est comme si on n'entrevoyait pas la possibilité d'éliminer le système patriarcal, alors que ce qui est construit peut être déconstruit. C'est là où je me définis comme féministe radicale. Je sais que cela n'arrivera sans doute pas de mon vivant et peut-être pas du vôtre non plus, mais pour moi, il faut vraiment changer fondamentalement la structure patriarcale et donc ne plus donner raison aux normes et aux idéologies qui la construisent. Ce n'est pas nécessairement en faisant une révolution, ça ne s'abat pas une structure patriarcale, ce n'est même pas comme le capitalisme, ça ne se voit pas. Ça se sent et ça se vit, par contre! Comme le signifiait Kate Millet en 1970, être radicale, c'est s'attaquer à la racine du mal. C'est pour cela que je me définis comme une féministe radicale matérialiste, cette racine se trouvant dans l'utilisation des femmes, de la matérialité de leur corps, de leur travail et de leur maternité, tout comme dans l'idéologie qui les construit et qui les situe dans un rapport inégalitaire.

Et pourquoi je me dis consubstantielle ou encore pourquoi j'adopte une perspective intersectionnelle? Parce que je crois fondamentalement maintenant - ce qui ne pourrait pas être lu dans les textes que j'écrivais au début des années 1970 - que les rapports sociaux de sexe ne se construisent jamais seuls. Le patriarcat a certes une relative autonomie, mais il existe dans une intersection, dans un enchevêtrement perpétuel et dynamique avec les autres rapports sociaux. On ne peut se contenter de regarder les rapports sociaux de sexe de manière univoque. Il faut même accepter que dans l'expérience d'une personne, le rapport de sexe ne soit pas toujours le rapport dominant dans des situations données, comme l'a démontré Danièle Kergoat. L'idée que je souhaite faire valoir quand je me dis consubstantielle est que tous les systèmes d'oppression s'alimentent, s'autoproduisent, s'interpénètrent dans un mouvement constant. Aussi, si je suis convaincue que le féminisme porte une proposition universelle et a permis d'améliorer la situation d'un grand

10. "Francine Descarries", Sociologues et sociologies québécoises (nouvelle série), mai 2019. Accessible sur le site du service audiovisuel de l'UQAM. 
nombre de femmes, et pas simplement celles de la classe des femmes privilégiées, il est tout aussi évident que la perspective consubstantielle favorise l'ouverture de l'analyse féministe à l'ensemble des processus sociaux et à leurs effets combinés, socialement et historiquement marqués. Elle ouvre sur le développement d'un vocabulaire et d'une pratique capables d'accueillir toutes les femmes et de faire place aux différences et disparités entre elles, aux tensions et positions qui les divisent, comme à leurs dilemmes identitaires au regard des conjonctures et des conditions matérielles et spatio-temporelles dans lesquelles elles évoluent.

Selon le moment, selon le contexte, ma pensée féministe peut faire appel aux concepts forts de ces différents courants de pensée et je peux adhérer à des stratégies qui sont développées par les unes et les autres, selon les circonstances et les objectifs poursuivis. Ce que je voulais donc dire par cette phrase, c'est que c'est une erreur, c'est encore un héritage des pratiques masculines que de vouloir s'inscrire dans une chapelle particulière. Il faut essayer de voir ce que chacune des chapelles peut nous offrir pour essayer de comprendre la réalité sociale dans toute sa complexité.

\section{Marie : Tu t'apprêtes à transmettre la direction du RéQEF. Quels sont les projets pour la suite?}

Essayer de passer une journée sans écrire un courrier électronique [rire]. Sérieusement... D’abord, je dois avouer que je ne pense pas être capable de me désintéresser du RéQEF du jour au lendemain, d'autant que je demeure dans son comité exécutif pour les prochaines trois années. Il occupera encore un certain temps dans mon agenda. Aussi, j'ai bien l'intention de reprendre en mains et de façon intensive la Ligne du temps de l'histoire des femmes au Québec ${ }^{11}$ que j'ai amorcée en 1985 avec mes premières étudiantes de l'UQAM et qui a été retravaillée pour sa diffusion sur le site du RéQEF. Je souhaite la revamper, l'enrichir et apporter plus de visibilité à la contribution des femmes dans leur diversité. Je m'aperçois que tout au long de ma carrière, j'ai voulu construire les archives et l'histoire du féminisme. D'ailleurs, au cours de l'année qui vient, j'organiserai deux colloques, l'un en sociologie et l'autre en études féministes, qui vont effectivement servir cet objectif. Dans les deux cas, il s'agit de profiter de l'événement du cinquantième anniversaire de l'UQAM pour essayer de construire une mémoire de l'institutionnalisation de ces deux champs de recherche. De plus, j'ai bien l'intention d'accorder le plus de temps possible à l'élaboration des derniers séminaires de maîtrise ou de doctorat que je donnerai, puisque je prendrai ma retraite de l'UQAM en 2021. Enfin, comme vous avez pu l'entendre, 
je sens le besoin de me ressourcer: cultiver des tomates ou écrire, on verra bien selon la situation à ce moment-là. J'aimerais beaucoup être capable de poursuivre ce travail de typologie que j'ai entrepris dès le début de ma carrière. Je m'aperçois, somme toute, que je suis une personne qui a eu une trajectoire assez rectiligne ou linéaire. Je n'ai pas tellement dérogé à mes intérêts théoriques et académiques. Je manque peut-être d'originalité! [Sourire.]

Véronica: Pour terminer cet entretien, peux-tu évoquer tes souhaits principaux pour les recherches féministes francophones à venir?

D'abord, qu'elles s'auto-fertilisent entre elles, qu'elles arrivent à faire valoir la grande qualité de la réflexion qui émerge à la fois de l'autre côté de l'Atlantique et ici, au niveau du genre, des rapports sociaux de sexe, du corps, de l'identité sexuelle. Au Québec, j'espère que les études féministes francophones vont pouvoir profiter de leur âge de maturité pour rayonner et imposer aux autres disciplines la perspective féministe. C'est bien beau de développer un bon programme en études féministes, mais quand on entend encore des professeurs dire: "Ce sujet-là sera traité en études féministes", cela nous force à réaliser qu'il reste encore du chemin à parcourir. Il faut briser cette résistance et convaincre nos collègues du bien-fondé des savoirs féministes et de leurs apports au savoir « universel ». Dans l'ensemble, je vois le développement des études féministes au Québec très positivement à condition qu'on garde le cap sur une logique de transformation sociale. Grâce aux différents CIRFF et aux contacts qui s'y développent, je constate qu'on travaille de mieux en mieux avec nos collègues françaises et cela devrait favoriser l'établissement de plus d'ententes, de cotutelles et d'échanges d'étudiant·e·s entre programmes. Il faudrait aussi penser à toute cette francophonie africaine qui est souvent laissée pour compte. Si nous nous nous jugeons victimes d'injustice épistémique, qu'en est-il pour elles? Si je considère essentiel que les études féministes demeurent un champ d'études spécialisé, il m'apparaît tout aussi indispensable qu'elles réussissent à pénétrer tous les espaces universitaires et tous les univers disciplinaires, que de plus en plus de chercheur·e·s se sentent obligé·e·s d'inscrire cette perspective dans leur réflexion et dans leur approche théorique. Bref, que les femmes ne soient pas toujours les éternelles oubliées de la réflexion théorique et des stratégies de développement. 